\title{
Solution scattering studies of the hierarchical assembly of porphyrin trimers based on benzene triscarboxamide
}

\author{
Richard van Hameren, ${ }^{a}$ Arend M. van Buul, ${ }^{a}$ Dirk Visser, ${ }^{b, c, d}$ Richard K. Heenan, ${ }^{c}$ Stephen M. King, \\ ${ }_{5}$ Alan E. Rowan, ${ }^{a}$ Roeland J. M. Nolte, ${ }^{a}$ Wim Pyckhout-Hintzen, ${ }^{*}$ Johannes A. A. W. Elemans ${ }^{{ }^{*} \mathrm{a}}$ and \\ Martin C. Feiters ${ }^{* a}$ \\ Received (in $X X X, X X X) X$ th $X X X X X X X X X 200 X$, Accepted $X$ th $X X X X X X X X X 200 X$ \\ First published on the web $X$ th $X X X X X X X X X 200 X$ \\ DOI: $10.1039 / b 000000 x$
}

10 The self-assembly of achiral and chiral porphyrin trimers based on benzene triscarboxamide in solution is studied with the help of NMR, FT-IR, UV-vis, and CD spectroscopy. These studies revealed that in apolar solvents the porphyrin trimers self-assembled in columnar stacks via a combination of hydrogen bonding and $\pi-\pi$ stacking interactions. While the critical aggregation constant is about $0.2 \mathrm{mM}$ in chloroform, aggregation already occurs at micromolar concentrations 15 in $n$-hexane. Small angle neutron scattering (SANS) studies in chloroform, toluene, and $n$-hexane confirmed aggregation of the trimers into columnar stacks. In chloroform and $n$-hexane, but not in toluene, the trimers gelated the solvent. In chloroform the stacks of the achiral trimer were found to contain on average about 70 molecules, while in toluene the stacks were much smaller and contained on average 7-9 molecules. In $n$-hexane the SANS studies revealed that the chiral trimer

20 formed a gel with an average mesh size of the transient network of chains of approximately $90 \mathrm{~nm}$, with chains being built up from effective cylindrical aggregates with an average length of $20 \mathrm{~nm}$, taking a screening or correlation length parameter into account in the simulation.

\section{Introduction}

An important goal in chemistry is to obtain control over the 25 organisation of molecular, macromolecular and supramolecular materials, with the objective to define or improve their function. ${ }^{1}$ In particular the controlled arrangement of chromophores into ordered self-assembled arrays is an evolving area of research with potential industrial 30 applications. ${ }^{2}$ It is envisioned that such arrays can yield new materials with unique photophysical, (opto)electronic and catalytic properties, as a result of the excitonic interactions between adjacent chromophoric moieties. ${ }^{3}$ Such supramolecular systems require the structural organisation and ${ }_{35}$ integration of a specific function. ${ }^{4}$ In addition, their arrangement in one (e.g. fibres), two (e.g. layers) or three (e.g. crystals) dimensions is required. One of the current challenges is to rationally design the shape of the molecular building blocks and tune the intermolecular interactions in such a way 40 that they spontaneously assemble into a supramolecular device.

For the creation of one-dimensional (1D) assemblies, building blocks with either a disk-like ${ }^{5}$ or a rod-like shape are typically employed. ${ }^{6}$ Also $1 \mathrm{D}$ assemblies composed of more ${ }_{45}$ complex building blocks have been reported, e.g. of circular macro-molecules, ${ }^{7}$ rosettes, ${ }^{8}$ and self-assembled dendrimers. ${ }^{9}$ We have previously described ${ }^{10}$ the self-assembly of porphyrin trimers 1 and 2 (Fig. 1), based on benzene triscarboxamide (BTA), ${ }^{11}$ into helical columnar stacks, which ${ }_{50}$ are stabilised by a combination of threefold hydrogen bonding between the amide functions and $\pi-\pi$ stacking interactions between the porphyrin planes. More recently, we also prepared and studied a set of analogues of $\mathbf{1}$ and $\mathbf{2}$ lacking 3 of the 4 phenyl groups in the meso-position of each porphyrin. ${ }^{12}$ ${ }_{55}$ The achiral porphyrin trimer $\mathbf{1}$ self-assembles into a racemate of $P$ - and $M$-helical stacks, whereas the chiral enantiopure trimer 2 yields stacks with one helical sense. ${ }^{13}$ Scanning tunneling microscopy (STM) and Atomic force microscopy (AFM) revealed that when micromolar solutions of these 60 compounds in chloroform were dropcasted onto a mica surface, extended patterns of equidistant single columnar stacks of molecules were obtained, which formed spontaneously as a result of a fine balance between molecular self-assembly and dewetting. ${ }^{11}$ In more apolar solvents like $n$ 65 hexane or cyclohexane, self-assembly of the trimers appeared to be much stronger, resulting in the formation of stacks in solution already at very low concentrations $\left(<10^{-8} \mathrm{M}\right)$. In that case, the assemblies are not in equilibrium with the monomeric building blocks, ${ }^{12}$ and dropcasting of their 70 solutions onto mica gave irregular networks of fibers with the width of a single molecule and lengths in the order of micrometers. ${ }^{13}$

Here we study in detail the aggregation behaviour of porphyrin trimers $\mathbf{1}$ and $\mathbf{2}$ based on BTA in different solvents 75 and with various techniques, including nuclear magnetic resonance (NMR), Fourier-transform infrared (FT-IR) spectroscopy, ultraviolet-visible (UV-vis) light spectroscopy, circular dichroism (CD), and small angle neutron scattering (SANS). We report on the shape and size of the assemblies 80 under various conditions, and on the hierarchical assembly of the columnar stacks of porphyrin trimers into networks that gelate the solvent. 

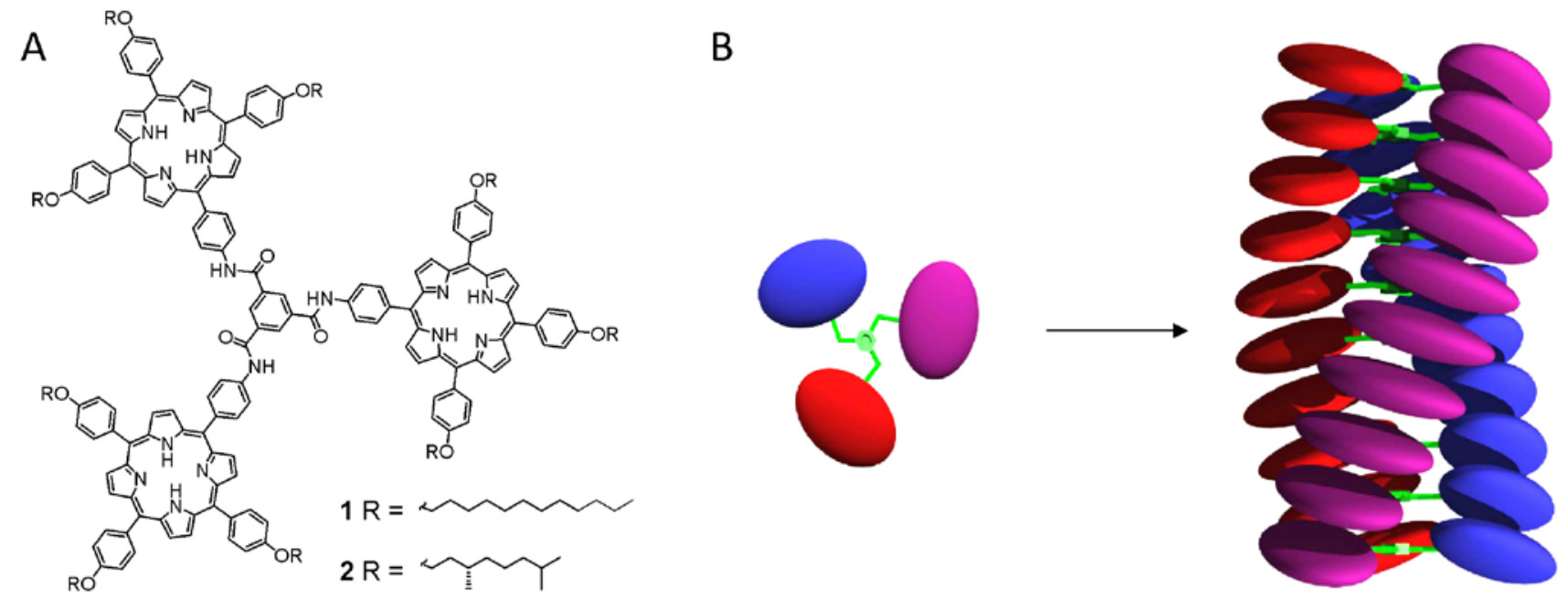

Fig. 1 (A) Molecular structures of the porphyrin trimers based on BTA. (B) Schematic representation of the self-assembly of porphyrin trimers into helical columnar stacks.

\section{${ }_{5}$ Results and Discussion}

\section{NMR spectroscopy}

In apolar solvents such as $\mathrm{CD}_{2} \mathrm{Cl}_{2}, \mathrm{CDCl}_{3}$, benzene-D6 and toluene-D8, the signals in the ${ }^{1} \mathrm{H}$ NMR spectra of $\mathbf{1}$ and $\mathbf{2}$ at millimolar concentrations were very broad, indicating 10 aggregation of these compounds. In Fig. 2 the ${ }^{1} \mathrm{H}$ NMR spectra of 1 in toluene-D8 without (bottom) and with a small droplet of DMSO-D6 (top) are shown. The top spectrum has sharp lines and indicates a breaking up of the aggregates by the presence of the polar solvent, probably by dissociation of 15 the hydrogen bonding network. The critical aggregation concentrations of $\mathbf{1}$ and $\mathbf{2}$ in $\mathrm{CDCl}_{3}$ were estimated to be $0.2 \pm$ $0.05 \mathrm{mM}$, since at that concentration broadening of the signals in the NMR spectra started to occur (ESI Fig. S1). In nhexane-D14, the porphyrin trimers gave broad NMR spectra 20 (not shown), even at concentrations of $0.05 \mathrm{mM}$, which indicates that in that solvent these compounds have a high tendency to aggregate.

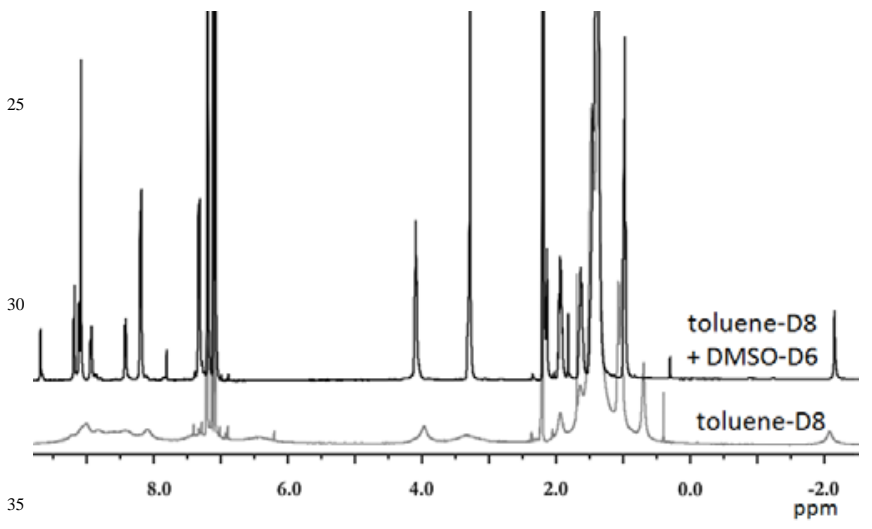

Fig. $2{ }^{1} \mathrm{H}$ NMR spectra of 1 in toluene-D8 (bottom) and in toluene-D8 with a droplet of DMSO-D6 (top) (400 MHz, $298 \mathrm{~K}$, [1] = $1 \mathrm{mM})$.

\section{FT-IR spectroscopy}

The role of hydrogen bonding in the aggregation of $\mathbf{1}$ was
40 investigated with Fourier transform infrared spectroscopy (FT-IR). Both in chloroform solution and in a dried film, the amide $\mathrm{N}-\mathrm{H}$ stretching vibration of this compound turned out to be very broad and overlapping with the pyrrole $\mathrm{N}-\mathrm{H}$ vibration, making an accurate assignment of the former ${ }_{45}$ impossible. However, the amide I and amide II stretching vibrations could be assigned, despite some interference with the porphyrin $\mathrm{C}=\mathrm{N}$ stretching vibration. The locations of the amide vibrations in chloroform solution and a dried film are summarised in Table 1. Both vibrations are located at rather 50 high frequencies, which is in accordance with the presence of a fully aromatic periphery. ${ }^{14}$ The position of the bands in the dried film is indicative of the presence of hydrogen bonding. The $\Delta v$-value, the difference in frequency between $v$ amide(I)

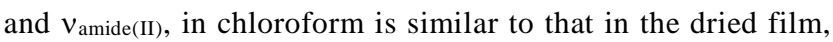
55 which indicates that comparable intermolecular hydrogen bonding interactions are present between the trimer molecules both in solution and in the solid state.

Table 1 FT-IR data of amide-related vibrations of 1.

\begin{tabular}{cccc}
\hline & & & \\
\hline Sample & $v_{\text {amide(I) }}\left(\mathrm{cm}^{-1}\right)$ & $v_{\text {amide(II) }}\left(\mathrm{cm}^{-1}\right)$ & $\Delta v\left(\mathrm{~cm}^{-1}\right)$ \\
\hline Dried film & 1662 & 1570 & 92 \\
$\mathrm{CHCl}_{3}$ solution & 1679 & 1588 & 91 \\
$(0.5 \mathrm{mM})$ & & & \\
\hline
\end{tabular}

\section{UV-vis and CD spectroscopy}

${ }_{60}$ The porphyrin Soret bands in the UV-vis spectra of $\mathbf{1}$ and $\mathbf{2}$ in chloroform at micromolar concentrations appeared sharp, indicating that the trimers are molecularly dissolved (Fig. $3 \mathrm{~A})$. In contrast, in $n$-hexane the spectra were significantly broadened (Fig. 3B). The Soret bands are split, and 65 deconvolution (not shown) revealed a red-shifted and a blueshifted component for both compounds. The broadening and the splitting of the Soret band are indicative of the presence of excitonically coupled neighbouring porphyrins. The Soret band splitting suggests relative orientations of the porphyrins 70 in both head-to-tail and face-to-face geometries. Compared to the spectrum of $\mathbf{1}$, the spectrum of $\mathbf{2}$ had a more blue-shifted 
A

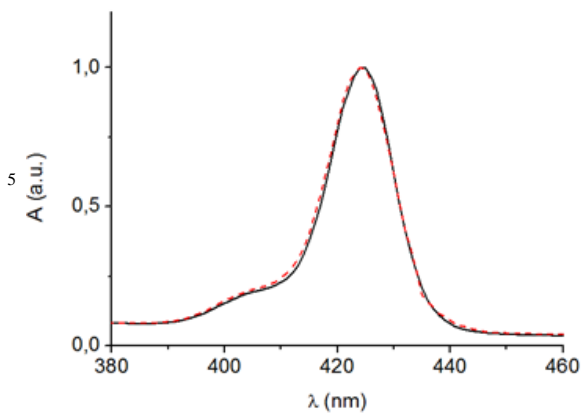

B

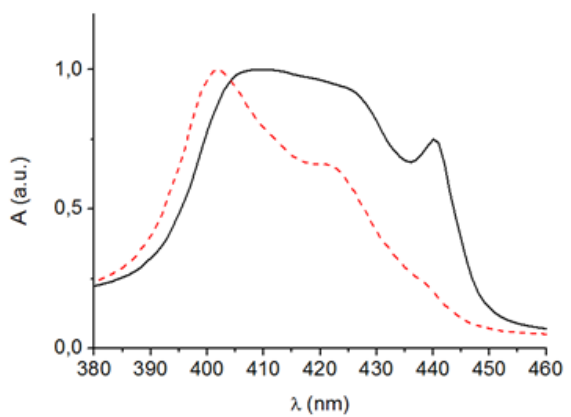

C

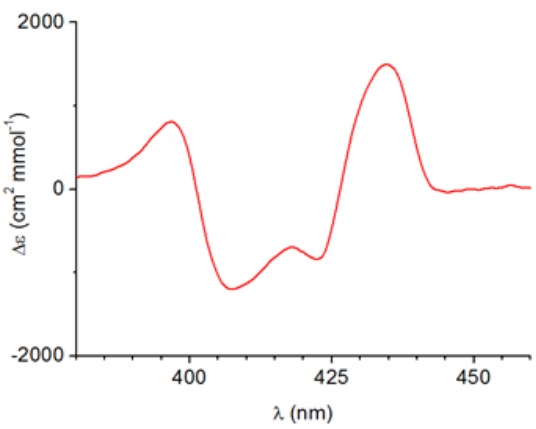

${ }_{10}$ Fig. 3 (A) UV-vis spectra (Soret band region) of $\mathbf{1}$ (solid black trace) and $\mathbf{2}$ (red dashed trace) in chloroform. (B) Idem, in $n$-hexane. (C) CD spectrum of 2 in $n$-hexane.

component, while its red-shifted component appeared as a shoulder. Apparently, the introduction of more steric bulk in the alkyl chains of 2 results in a preference for face-to-face 15 orientation of the porphyrins in the self-assembled structure. The UV-vis spectra of $\mathbf{1}$ and $\mathbf{2}$ in $n$-hexane indicate intermolecular aggregation of these compounds, since intramolecular stacking of the porphyrins would impose too much strain on the molecules. Since plots of the absorbance at 20 various wavelengths versus the concentration revealed Lambert-Beer behaviour over a wide concentration range, it is proposed that in $n$-hexane both compounds are arranged in a well-defined self-assembled structure.

In the case of 2, circular dichroism (CD) studies confirmed 25 the intermolecular aggregation suggested by the UV-vis studies. While no CD-effects were observed in chloroform at micromolecular concentrations, the CD spectrum in $n$-hexane clearly indicated the presence of chiral assemblies (Fig. 3C). ${ }^{12}$ Deconvolution of the spectrum revealed the existence of three ${ }_{30}$ Cotton effects centered at 402, 426 and $438 \mathrm{~nm}$, respectively. As expected, the achiral compound $\mathbf{1}$ did not show any detectable CD-effect (not shown), in line with the literature result ${ }^{12}$ for its analogue lacking most of the phenyl groups in the meso-positions of the porphyrins.

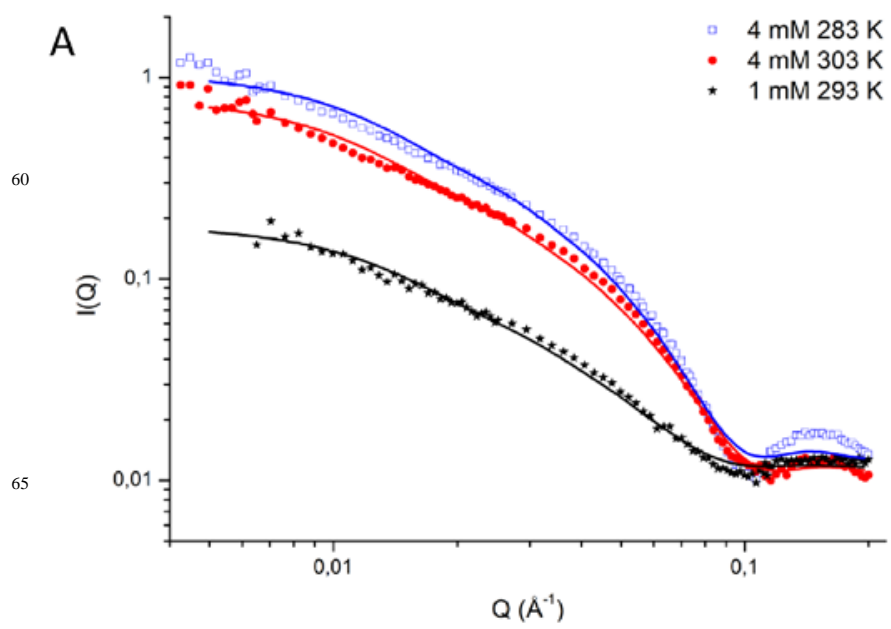

35

\section{Small Angle Neutron Scattering (SANS)}

The NMR, FT-IR, UV-vis and CD studies all indicate aggregation of the molecules in $\mathbf{1}$ and $\mathbf{2}$ in organic solvents. In line with other self-assembled structures of disk-shaped 40 molecules based on BTA, ${ }^{5 a}$ it can be expected that the porphyrin trimers self-assemble into columnar stacks. To test this hypothesis, SANS studies were carried out on the assemblies under a variety of experimental conditions.

The critical aggregation concentration of $\mathbf{1}$ in chloroform is 45 approximately $0.2 \mathrm{mM}$. When the concentration was increased beyond this value, the solution rapidly became more viscous and gelated at a concentration of approximately $2 \mathrm{mM}$. These observations indicate strong aggregation of the molecules. The shape and dimensions of the aggregates were determined 50 by SANS. The scattering intensity profile i.e. the dependency on the scattering vector $\mathrm{Q}$ of $\mathbf{1}$ in $\mathrm{CDCl}_{3}$ at $283 \mathrm{~K}([\mathbf{1}]=4$ $\mathrm{mM}$ ) showed a clear $\mathrm{Q}^{-1}$ dependence between 0.008-0.03 $\AA^{-1}$, which is characteristic for a $1 \mathrm{D}$ object, and a pronounced minimum at $\mathrm{QR} \sim 3.83$, which together revealed the presence 55 of cylindrical rod-shaped particles with length $\mathrm{L}$ and spherical cross section of radius $\mathrm{R}$ (Fig. 4A).

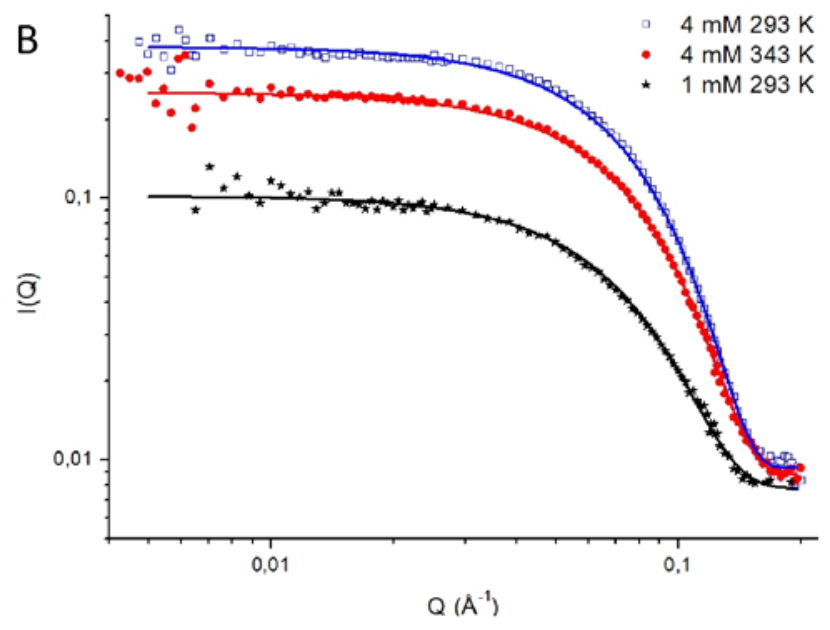

Fig. 4 (A) Scattering intensity profile of 1 in $\mathrm{CDCl}_{3}$ at various temperatures and concentrations. (B) Idem, in toluene-D8. The solid lines represent fits assuming a theoretical model based on a homogeneous cylindrical rod. 


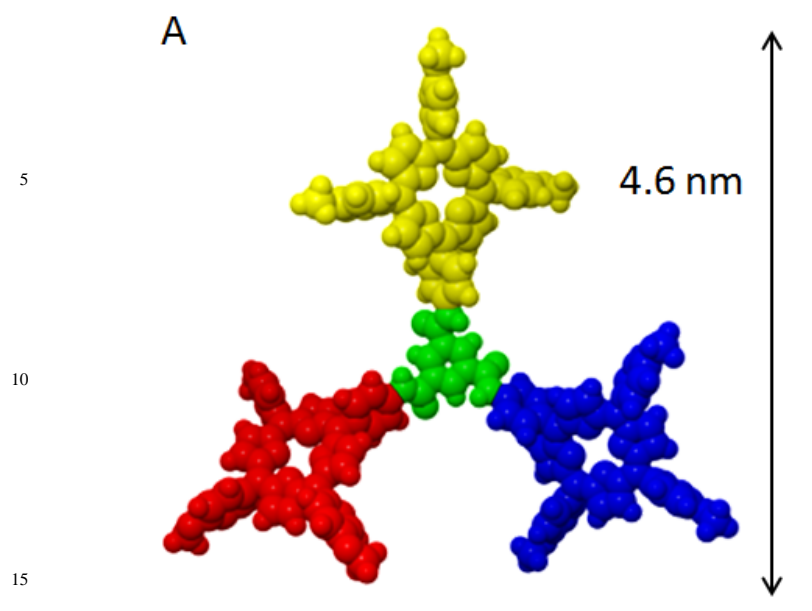

B

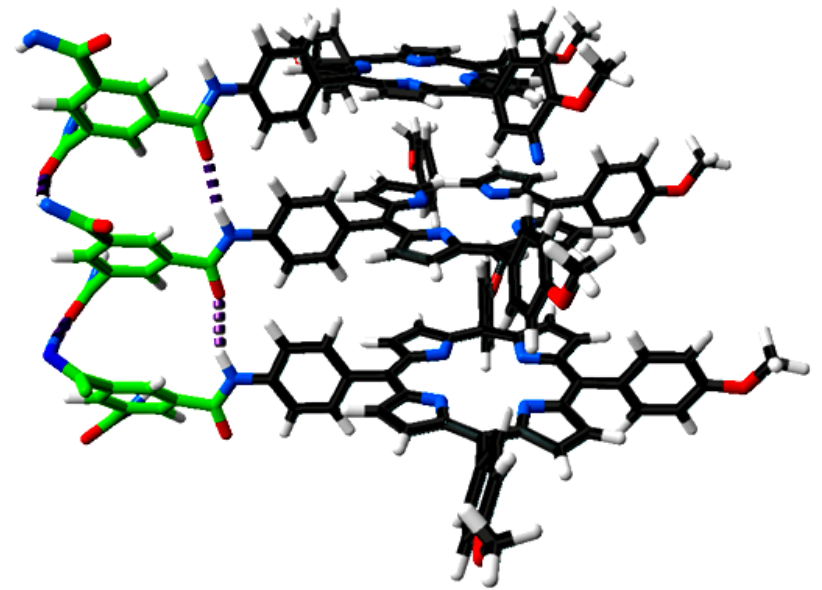

Fig. 5 (A) Space-filling molecular model of the aromatic core of $\mathbf{1}$ and 2; alkyl tails have been omitted for clarity. (B) Molecular model of the intermolecular hydrogen bonding network between molecules of $\mathbf{1}$ or $\mathbf{2}$; only one porphyrin moiety is shown for clarity, and alkyl tails have been omitted.

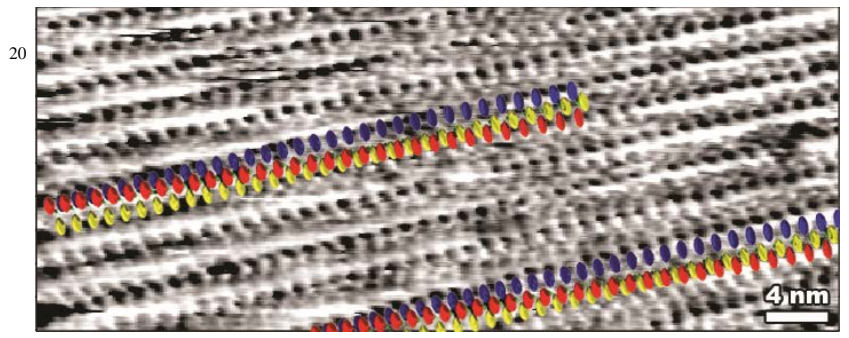

25 Fig. 6 STM image of a self-assembled monolayer of $\mathbf{1}$ at the interface of Highly Ordered Pyrolytic Graphite (HOPG) and 1-phenyloctane,

$\mathrm{V}_{\text {bias }}=-369 \mathrm{mV}, \mathrm{I}_{\text {set }}=1.0 \mathrm{pA}$. Schematic models of $\mathbf{1}$ are superimposed on some parts of the STM image.

When a different model was applied, e.g. assuming a so sphere, no plausible fit whatsoever could be obtained. The data could be simulated with a theoretical model (see ESI) based on a homogeneous cylindrical rod with a radius $(\mathrm{R})$ of $3.5 \pm 0.1 \mathrm{~nm}$ and a length (L) of $43 \pm 2 \mathrm{~nm}$. Molecular modeling calculations of a columnar stack of $\mathbf{1}$ revealed that 35 the radius of its aromatic core is $2.3 \mathrm{~nm}^{10}$ (Fig. 5). Its dodecyl tails have a length of $1.4 \mathrm{~nm}$ in the case they are fully stretched. Taking these values into account, it is plausible to describe the particles in solution as columnar stacks that are each a single molecule thick. Their 40 determined length indicates that on average one columnar stack consists of approximately 70 molecules, assuming an intermolecular distance of $0.6 \mathrm{~nm}$ which followed from the molecular modelling calculations and which was confirmed by STM measurements of stacks of $\mathbf{1}$ on a graphite surface ${ }_{45}$ (Fig. 6). ${ }^{10}$

When the temperature of the solution was increased, SANS revealed a significant decrease in the average length of the cylinder between 293 and 303 K (Table 2 and Fig. $4 \mathrm{~A}$ ), while the radius remained constant, indicating a 50 decrease in the number of molecules within a single columnar stack. Lowering the concentration resulted in an even more pronounced decrease in average stack length, while the average diameter remained roughly constant.
Table 2 SANS fitting results and errors for 1 in $\mathrm{CDCl}_{3}$ and toluene-D8 55 at various temperatures and concentrations.

\begin{tabular}{ccccc}
\hline Solvent & T (K) & $\begin{array}{c}\text { Concentration } \\
(\mathrm{mM})\end{array}$ & $\begin{array}{c}\text { Rod } \\
\text { length } \mathrm{L} \\
(\mathrm{nm})\end{array}$ & $\begin{array}{c}\text { Rod } \\
\text { radius R } \\
(\mathrm{nm})\end{array}$ \\
\hline $\mathrm{CDCl}_{3}$ & 283 & 4.0 & $43 \pm 2$ & $3.5 \pm 0.1$ \\
& 293 & 4.0 & $45 \pm 2$ & $3.5 \pm 0.1$ \\
& 303 & 4.0 & $34 \pm 2$ & $3.4 \pm 0.1$ \\
& 293 & 2.0 & $40 \pm 4$ & $3.7 \pm 0.1$ \\
& 293 & 1.0 & $25 \pm 2$ & $3.4 \pm 0.1$ \\
Toluene- & 293 & 4.0 & $5.3 \pm 0.1$ & $2.3 \pm 0.1$ \\
D8 & & & & \\
& 318 & 4.0 & $5.5 \pm 0.1$ & $2.2 \pm 0.1$ \\
& 343 & 4.0 & $5.4 \pm 0.1$ & $2.2 \pm 0.1$ \\
& 293 & 2.0 & $5.8 \pm 0.1$ & $2.3 \pm 0.1$ \\
& 293 & 1.0 & $6.3 \pm 0.2$ & $2.2 \pm 0.1$ \\
\hline
\end{tabular}

Also in a solution of $\mathbf{1}$ in toluene-D8 ([1] = $4 \mathrm{mM})$ SANS experiments indicated the presence of aggregates (Fig. 4B). At $293 \mathrm{~K}$ the scattering profile could still be fitted to a 60 cylindrical rod, with a radius of $2.3 \mathrm{~nm}$ and a length of 5.3 $\mathrm{nm}$ (Table 2); it should be noted that, compared to the results for $\mathrm{CDCl}_{3}$ in Fig. 4A, the characteristic $\mathrm{Q}^{-1}$ dependence of the rod is lost, however, due to the lower aspect ratio $\mathrm{L} / \mathrm{R}$, but the dip in the form factor is still 65 recognized. The radius is again in good agreement with the modelled radius of the aromatic part of the molecule, while the determined length corresponds to columnar stacks that contain on average 9 molecules. The difference in length between the assemblies in chloroform and toluene 70 underlines the importance of $\pi-\pi$ interactions in the formation of the columnar stacks; since toluene, unlike chloroform, is itself aromatic, it interferes with the $\pi-\pi$ interactions between the aromatic moieties in the molecules of $\mathbf{1}$. The relatively small size of the assemblies is further in 75 agreement with the observation that a $4 \mathrm{mM}$ solution of 1 in toluene-D8 is not gelated at all, i.e. the gelation must be correlated with the presence of rather stiff entities. The gelation is also reflected in different effects for the alkyl tails. In chloroform, they are involved in gelation of the 
solvent, and contribute to a relatively rigid outer shell of the cylindrical rod, consisting of alkyl tails and solvent molecules; this contributes to the scattering length density contrast, and a relatively large apparent radius $(3.5 \mathrm{~nm})$. In 5 toluene the sparse alkyl tails are 'dissolved', resemble the solvent much more in dynamics, and only contribute in a minor way to the scattering intensity, resulting in a much smaller apparent radius $(2.3 \mathrm{~nm})$ for the aggregate, which corresponds to that of the aromatic core. Considering the 10 disintegration of the same system in chloroform at higher temperature and lower concentration, it is remarkable that the aggregates of $\mathbf{1}$ in toluene turned out to be very stable. At elevated temperatures $(\mathrm{T}=318$ and $343 \mathrm{~K}$ ), no significant change in their dimensions was observed (Fig.

15 4B, Table 2). Also a 2- and 4-fold dilution of the sample resulted in only small changes in the dimensions of the volume of the rods. It should be noted that the scattering profile becomes less pronounced upon dilution because of the reduction of intensity due to the lower number of 20 scatterers and their respective size. The forward scattering intensities in both solvents at $\mathrm{Q}=0$ at fixed concentration are in full agreement with the different contrasts $(\sim 4$ times higher in D8-Toluene) and the scattering aggregate volumes, derived from the parameters, obtained from fitting 25 the full Q-dependence. These all are strong arguments that single individual rod-like assemblies are predominantly present and a structure factor due to interaction between species can be safely neglected $(S(Q)=1)$.

A solution of 2 in $n$-hexane- $\mathrm{D}_{14}([2]=4 \mathrm{mM})$ also 30 gelated. Assuming that also here assemblies with a cylindrical shape are present, the best fit of the SANS data was obtained by assuming a rod with length of $53.1 \pm 0.3$ $\mathrm{nm}$ and a radius of $2.18 \pm 0.01 \mathrm{~nm}$ (Fig. 7). Contrary to the experiments described above for $\mathrm{CDCl}_{3}$ or D8-Toluene, the 35 experimental error bars of the data for the low $Q$-values $(Q$ $<5 \times 10^{-3} \AA^{-1}$ ) were relatively small and hence the data in this range are statistically relevant; at these low $Q$-values this model does not describe the data very well due to a clear upturn of the intensity following a $\mathrm{Q}^{-2}$ dependence. $40 \mathrm{An}$ onset of the same behaviour though of much lower extent, could also be suspected in the $\mathrm{CDCl}_{3}$ data, in which the cylindrical length $(\sim 50 \mathrm{~nm})$ is comparable. In Toluene no sign of it appears, however, as expected on the basis of the low aspect ratio in this case. In a typical SANS 45 measurement of dilute particles as described above, all mutual interactions between the particles can be neglected, and the contribution of the inter-particle structure factor $S(Q)$ is taken as 1 . In a gel, such mutual interactions are obviously and inherently present. Simple structure factors

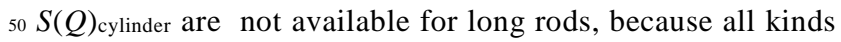

of orientation issues need to be addressed, e.g. the amount of alignment and the rate of entanglement of the rods. In order to account for the upturn at low Q, we followed the approach of Pezron et al., who demonstrated that it is 55 possible to make an approximation of the structural parameters of solutes in a mesh, by taking into account the so-called screening or correlation length $\xi .{ }^{15}$ With this $\xi$ value it is possible to express the structural parameters of solutes in a mesh as a function of those in a dilute solution.

60 In a good solvent for the rods, the $\xi$-value corresponds to the average mesh-size in a transient network of chains. When the SANS data of $\mathbf{2}$ in $n$-hexane-D14 were fitted with this model, an improved fit was obtained with a cylinder of radius of $2.3 \pm 0.05 \mathrm{~nm}$ and an effective length of approx. $6520 \mathrm{~nm}$ (Fig. 7). The $\xi$-value could not be determined very accurately; a value of $90 \pm 25 \mathrm{~nm}$ was estimated and a correlation of latter value with the length of the rod can be expected. The relatively large error in the $\xi$-value reflects the lack of extended data at lower Q. Inclusion of 70 polydispersity, in particular in the rod length and/or allowance for flexibility in the rod could improve the fit at low $\mathrm{Q}$ as well, but introduces more fitting parameters than is justified by the data. As the rod length approaches the mesh size a more detailed 3-dimensional simulation would 75 be required. The radius of the cylinder is in good agreement with the radius of the aromatic plane of 2 , which confirms that in the $n$-hexane gel columnar stacks of $\mathbf{2}$ are present; this is also in line with the results of static light scattering experiments on more dilute samples, ${ }^{12}$ where, however, the 80 particles appeared to be much larger (length $300 \pm 50 \mathrm{~nm}$, radius of gyration of the order of $90 \mathrm{~nm}$ ). In the simulation of the SANS of the gel of $\mathbf{2}$ in $n$-hexane, the alkyl tails do not appear to contribute to the cylinder radius, which is identical to the observation to the aggregates of $\mathbf{1}$ in toluene, ${ }_{85}$ but different from the gel of $\mathbf{1}$ in chloroform (Table 1); this difference could point to a different role for the alkyl tails in the gels in chloroform and $n$-hexane, but could also be due to the lack of contrast between the alkyl tails and $n$-hexane. According to the cylinder-in-mesh model, the gel has an 90 average mesh size of the transient network of chains of approximately $90 \mathrm{~nm}$ and these chains are built up from cylindrical aggregates. This compares well to the estimate of the diagonal which is $\mathrm{L} \sqrt{3}$ with $\mathrm{L} \sim 50 \mathrm{~nm}$. The average length of approximately $20 \mathrm{~nm}$ found for these cylinders in 95 the Pezron-modified model represents a persistence length or subunit length of inflexible cylinder fragments, as opposed to the more rigid cylinders with a length of approximately $53 \mathrm{~nm}$ found upon simulation with the cylindrical rod model alone (see above).

100 


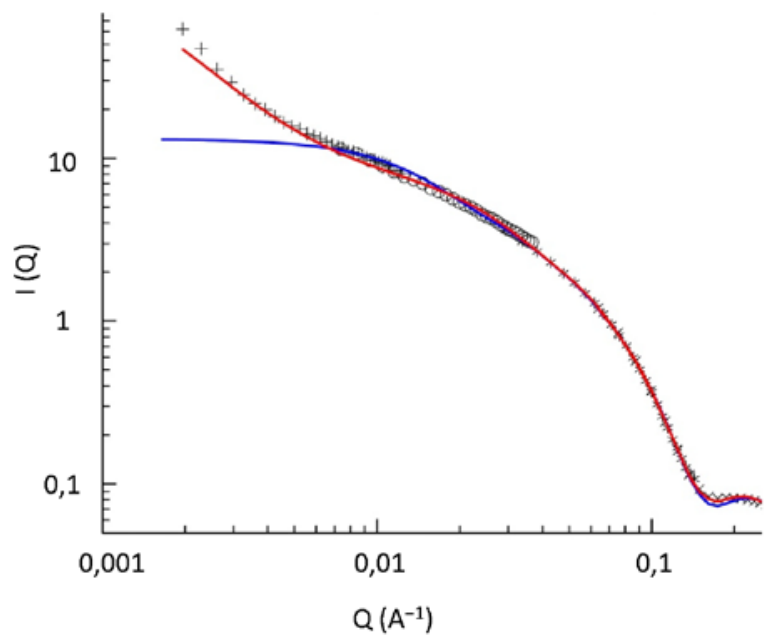

Fig. 7 Scattering intensity profile of 2 in $n$-hexane-D14 (crosses, [2] = $4 \mathrm{mM}$ ) at $293 \mathrm{~K}$ and the fitted theoretical models (blue, cylinder; red, cylinder in a mesh).

Intuitively, a gel is expected to be a hierarchically built-up 5 assembly containing very long, entangled fibres, such as obtained by solvent evaporation from the gel in the aforementioned AFM study, which also contains an artist's impression of the interaction of the fibers in the gel. ${ }^{13}$ However, the SANS measurements indicated the presence 10 of only relative small columnar aggregates (approximately 70 molecules per stack) in both the gels of 1 in $\mathrm{CDCl}_{3}$ and of $\mathbf{2}$ in $n$-hexane-D14. Static light scattering experiments of the assemblies of $\mathbf{2}$ in $n$-hexane previously suggested the presence of rod-like particles with an average length of 300 ${ }^{15} \mathrm{~nm},{ }^{12}$ a much larger value than derived for the length of the columnar stacks from the SANS data of 2 in $n$-hexane-D14. Although the static light scattering result was obtained on much more dilute solutions, and was analysed without consideration of the shape of the aggregate, and dimensions 20 are generally somewhat overestimated by light-scattering, ${ }^{16}$ the discrepancy between the stack sizes of $\mathbf{2}$ determined by the two techniques is very large in this case. The difference likely originates from the difference in Q-range. Whilst light scattering techniques yield information about the 25 global dimensions of the assemblies, SANS studies yield more information about the local organisation within the assemblies. It should also be noted that the plateau or Guinier regime, which allows an independent estimation of the radius of gyration $R_{\mathrm{g}}$ (and from there the cylinder length 30 $\mathrm{L}$ via the relation $\mathrm{Rg}^{2}=\mathrm{L}^{2} / 12$ from the relation $\mathrm{Q} R_{\mathrm{g}}<1$ ), can be just detected in the SANS pattern of the dispersion of $\mathbf{1}$ in toluene (Fig. 4B) but is not visible in those of the gels of 1 in chloroform (Fig. 4A) and of 2 in hexane (Fig. 7); However, $\mathrm{L}$ and $\mathrm{R}$ are both parameters of the whole form 35 factor and are obtained in good accuracy from the Qdependence. Nevertheless, the cylinder lengths L derived from the SANS simulations should probably be considered as lower limits. As a result, the SANS-derived dimensions of the cylindrical assemblies of $\mathbf{1}$ in $\mathrm{CDCl}_{3}$ and 2 in $n$ 40 hexane-D14 obtained by SANS can be interpreted as a sort of persistence length or subunit length of these assemblies. A similar approach was taken in SANS studies of oligo( $p$ - phenylenevinylene)s, where the upturn at low $\mathrm{Q}$ was taken as evidence of entanglement of smaller units, for which the 45 length found by SANS corresponded to the persistence length as derived from AFM. ${ }^{17}$ In a study on the behaviour of poly(benzyl methacrylate) in ionic liquids, the upturn was interpreted as due to the coexistence of dispersed polymer chains and larger clusters. ${ }^{18}$ Especially since the 50 SANS measurements were performed in a gel and a solidcylinder model was applied to describe the data, such an interpretation seems justified. On the contrary, a worm-like description of the scattering intensities seems inappropriate as it would defer the discussion of mesh sizes and the 55 corresponding macroscopic gelling of the samples to that of a single chain character of dilute semi-flexible chains in solutions that do not gel. The upturn of the intensity would then be associated with contributions of correlated distances exceeding those of the stiff segment length.

\section{${ }_{60}$ Experimental section}

\section{General}

Chloroform was distilled from $\mathrm{CaH}_{2}$ prior to use. $n$-Hexane was distilled over $\mathrm{Na}$ prior to use. NMR spectra were recorded on Bruker DPX-200, Bruker DMX-300 or Varian

65 Inova-400 instruments. Chemical shifts are reported in ppm downfield with respect to the solvent peak. FT-IR spectra were recorded on a ThermoMattson IR300 spectrometer equipped with a Harrick ATR unit and the compounds were measured as a solution or a film. UV-vis spectra were 70 recorded on a Varian Cary 50 Conc spectrophotometer at ambient temperature. CD spectra were recorded on a Jasco 810 instrument equipped with a Peltier temperature control unit. STM experiments were carried out in the constant current mode on a home-built setup by applying a droplet of

75 a solution of $\mathbf{1}$ in 1-phenyloctane to a freshly cleaved HOPG surface.

\section{Syntheses}

The syntheses of $\mathbf{1}^{10}$ and $\mathbf{2}^{12}$ have been described 80 previously.

\section{SANS experiments}

SANS experiments on $\mathbf{1}$ were performed at the KWS1 spectrometer at the FRJ-2 Dido Reactor at Jülich, ${ }_{85}$ Germany. ${ }^{19}$ The SANS experiments on 2 in $n$-hexane-D14 were performed at the small angle scattering instrument D11 at the Institute of Laue-Langevin in Grenoble, France. ${ }^{20}$

\section{Conclusions}

We have shown that in chloroform the amide-linked 90 porphyrin disks $\mathbf{1}$ and $\mathbf{2}$ self-assemble into well-defined columnar stacks as a result of hydrogen bonding and $\pi-\pi$ interactions, after exceeding a critical aggregation concentration (e.g. $0.2 \mathrm{mM}$ for 1). In $n$-hexane, a solvent in which the $\pi-\pi$ interactions are enhanced, the porphyrin 95 trimers self-assemble into columnar stacks at micromolar concentrations. In toluene the self-assembly is weakest and SANS studies revealed the presence of stacks of $\mathbf{1}$, resulting 
in cylindrical rods with a relatively short aspect ratio (radius $2.3 \mathrm{~nm}$, length $5.3 \mathrm{~nm}$, corresponding to a stack of approximately 7-9 trimers). In chloroform, stacks of $\mathbf{1}$ contain about 70 trimers (radius $3.5 \mathrm{~nm}$, length $43 \mathrm{~nm}$ ), a 5 number that decreases upon an increase in temperature or a decrease in concentration. In $n$-hexane, self-assembly is strongest and SANS studies on a gel of $\mathbf{2}$ and this solvent indicated that it had a transient network chain of approximately $90 \mathrm{~nm}$, built-up from cylindrical aggregates 10 of 2 with an average length of $20 \mathrm{~nm}$ (radius $2.3 \mathrm{~nm}$ ) within a specific approach for gels.

\section{Acknowledgements}

The authors thank Dr. Isabelle Grillo for supporting measurements at ILL. The National Research School ${ }_{15}$ Combination Catalysis (NRSC-C) (R.v.H.), the Royal Netherlands Academy of Science (R.J.M.N), and the Ministry of Education, Culture and Science (Gravity program 024.001.035) are acknowledged for financial support. J.A.A.W.E. wishes to thank the Council for the ${ }_{20}$ Chemical Sciences of the Netherlands Organisation for Scientific Research (CW-NWO) for a Vidi grant (700.58.423), the European Research Council for an ERC Starting Grant (NANOCAT - 259064). D. V. was supported by Physics-NWO. EP-SERC (UK) is acknowledged for the ${ }_{25}$ provision of beam time (experiment 9-11-1196) and support (D. V., S. M. K.) at the ILL. The Helmholtz Society (Germany) is acknowledged for the provision of beam time (experiments KW1-04-069 and KW1-05-052) and support at the Forschungszentrum Jülich; this research project has 30 been supported by the European Commission under the 6th Framework Programme through the Key Action: Strengthening the European Research Area, Research Infrastructures, contract $\mathrm{n}^{\circ}$ : RII3-CT-2003-505925.

\section{Notes and references}

35 a) Department of Organic Chemistry, Institute for Molecules and Materials, Faculty of Science, Radboud University Nijmegen, Heyendaalseweg 135, 6525 AJ, Nijmegen, The Netherlands. E-mail: m.feiters@science.ru.nl; Fax: +.31.24.3652929; Tel: $+.31 .24 .3652016 / 2676$

40 b) Large Scale Structures Group, ISIS Pulsed Neutron \& Muon Source, Science \& Technology Facilities Council, Rutherford Appleton Laboratory, Harwell Oxford, Didcot, OX11 0QX, United Kingdom c) NWO Physics, present address: Reactor Institute Delft, Department RRR, Section FAME, Delft University of Technology, Mekelweg 15,

452629 JB Delft, The Netherlands

d) Department of Physics, Loughborough University, Loughborough LE11 3TU, UK

e) Jülich Centre for Neutron Science-1 \& Institute for Complex Systems-1, Forschungszentrum Jülich GmbH, 52425 Jülich, Germany

50

† Electronic Supplementary Information (ESI) available: Critical aggregation constant determination of compound 2, details of SANS experiments at KWS-1, SANS theoretical model.. See DOI: $10.1039 / \mathrm{b} 000000 \mathrm{x} /$

55 ‡ Footnotes should appear here. These might include comments relevant to but not central to the matter under discussion, limited experimental and spectral data, and crystallographic data.
1 (a) S. D. Hudson, H. T. Jung, V. Percec, W. D. Cho, G. Johansson, G. Ungar and V. S. K. Balagurusamy, Science, 1997, 278, 449. (b) S. R. Forrest, Nature, 2004, 428, 911.

2 J. A. A. W. Elemans, R. van Hameren, R. J. M. Nolte and A. E. Rowan, Adv. Mat., 2006, 18, 1251.

3 M. D. Ward, Chem. Soc. Rev., 1997, 26, 365.

4 J.-M. Lehn. Proc. Natl. Acad. Sci. U.S.A., 2002, 99, 4763.

5 (a) A. R. A. Palmans and E. W. Meijer, Angew. Chem. Int. Ed., 2007, 46, 8948. (b) S. Kumar, Chem. Soc. Rev., 2006, 35, 83. (c) H. M. Keizer and R. P. Sijbesma, Chem. Soc. Rev., 2005, 34, 226.

6 (a) J. D. Hartgerink, E. Beniash and S. I. Stupp, Science, 2001, 294, 1684. (b) F. J. M. Hoeben, P. Jonkheijm, E. W. Meijer and A. P. H. J. Schenning, Chem. Rev., 2005, 105, 1491. (c) P. Jonkheijm, P. van der Schoot, A. P. H. J. Schenning and E. W. Meijer, Science, 2006, 313, 80.

7 (a) W. S. Horne, N. Ashkenasy and M. R. Ghadiri, Chem. Eur. J., 2005, 11, 1137. (b) S. Höger, Chem. Eur. J., 2004, 10, 1320.

8 R. S. Johnson, T. Yamazaki, A. Kovalenko and H. Fenniri, J. Am. Chem. Soc., 2007, 129, 5735.

9 (a) S. C. Zimmerman, F. Zeng, D. E. C. Reichert and S. V. Kolotuchin, Science, 1996, 271, 1095. (b) V. Percec, W. D. Cho, P. E. Mosier, G. Ungar and D. J. P. Yeardley, J. Am. Chem. Soc., 1998, 120, 11061.

10 R. van Hameren, P. Schön, A. M. van Buul, J. Hoogboom, S. V. Lazarenko, J. W. Gerritsen, H. Engelkamp, P. C. M. Christianen, H. A. Heus, J. C. Maan, Th. Rasing, S. Speller, A. E. Rowan, J. A. A. W. Elemans and R. J. M. Nolte, Science, 2006, 314, 1433.

11 S. Cantekin, T. F. A. de Greef and A. R. A. Palmans, Chem. Soc. Rev., 2012, 41, 6125

12 N. Veling, R. van Hameren, A. M. van Buul, A. E. Rowan, R. J. M. Nolte and J. A. A. W. Elemans, Chem. Commun. 2012, 48, 4371.

13 R. van Hameren, A. M. van Buul, M. A. Castriciano, V. Villari, N. Micali, P. Schön, S. Speller, L. Monsù Scolaro, A. E. Rowan, J. A. A. W. Elemans and R. J. M. Nolte, Nano Lett., 2008, 8, 253

14 L. J. Bellamy. The Infrared Spectra of Complex Molecules, Vol. Two (Chapman and Hall, London and New York, 1980).

15 I. Pezron, M. Djabourov and J. Leblond, Polymer, 1991, 32, 3201.

16 F. Ikkaia and M. Shibayama, Polymer, 2007, 48, 2387.

17 P. Jonkheijm, F. J. M. Hoeben, R. Kleppinger, J. van Herrikhuyzen, A. P. H. J. Schenning, and E. W. Meijer, J. Am. Chem. Soc., 2003, 125, 15941.

18 K. Fujii, T. Ueki, K. Niitsuma, T. Matsunaga, M. Watanabe, M. Shibayama, Polymer 2011, 52, 1589..

19 Http://www.fzjuelich.de/jcns/DE/Leistungen/Instruments2/Structures/KWS1/_no de.html

20 Http://www.ill.eu/instruments-support/instrumentsgroups/instruments/d11/description/instrument-layout/

Text of Table of Contents Graph:

'Columnar stacks are intermediates in the solvent-dependent selfassembly of benzene triscarboxamides to more highly organized structures such as gels' 